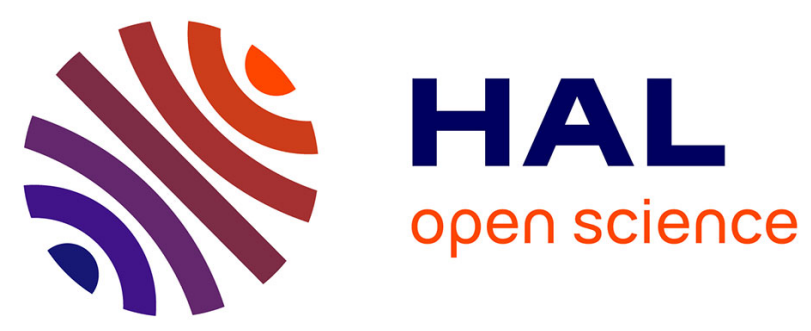

\title{
Transcatheter aortic valve replacement performed with selective telemetry monitoring: A prospective study
}

Mariama Akodad, Eissa Aldhaheri, Gregory Marin, François Roubille, Jean-Christophe Macia, Thomas Gandet, Delphine Delseny, Laurent Schmutz, Benoit Lattuca, Pierre Robert, et al.

\section{To cite this version:}

Mariama Akodad, Eissa Aldhaheri, Gregory Marin, François Roubille, Jean-Christophe Macia, et al.. Transcatheter aortic valve replacement performed with selective telemetry monitoring: A prospective study. International Journal of Cardiology, 2021, 330, pp.158-163. 10.1016/j.ijcard.2021.02.028 . hal-03151022

\section{HAL Id: hal-03151022 \\ https://hal.science/hal-03151022}

Submitted on 13 Jun 2021

HAL is a multi-disciplinary open access archive for the deposit and dissemination of scientific research documents, whether they are published or not. The documents may come from teaching and research institutions in France or abroad, or from public or private research centers.
L'archive ouverte pluridisciplinaire HAL, est destinée au dépôt et à la diffusion de documents scientifiques de niveau recherche, publiés ou non, émanant des établissements d'enseignement et de recherche français ou étrangers, des laboratoires publics ou privés. 


\section{Transcatheter aortic valve replacement performed with selective telemetry monitoring: A prospective study}

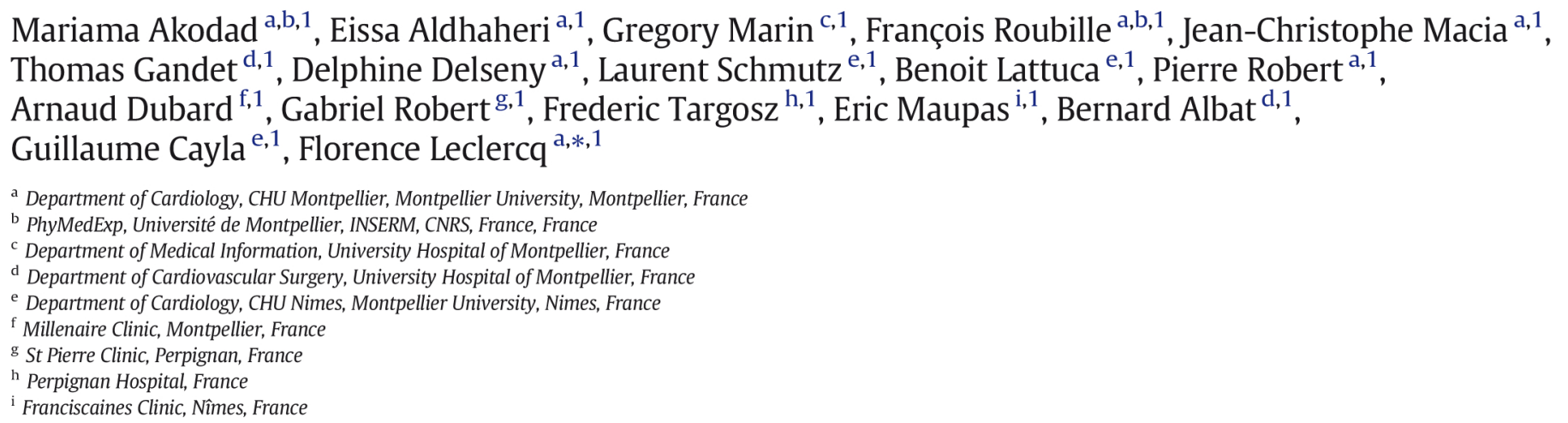

Keywords:

TAVR

Complications

Intensive care unit

Conductive disorders

Telemetry monitoring

\begin{abstract}
A B S T R A C T
Background: Telemetry monitoring (TM) with or without intensive care unit (ICU) admission is the standard of care after Transcatheter aortic valve replacement (TAVR). Regarding to improvements of the technique and procedural results, TM may be considered only in selected patients. We aimed to confirm feasibility and safety of selective TM in patients undergoing TAVR.

Methods: We prospectively evaluated 449 consecutive patients undergoing TAVR. Patients were transferred to general cardiology ward (GCW) without TM after the procedure when stable clinical state, transfemoral access, no baseline right bundle branch block (RBBB), left ventricular ejection fraction (LVEF) $>40 \%$, and no complication including any electrocardiogram (ECG) change within $1 \mathrm{~h}$ after the procedure ("low-risk" group). Others patients were considered for TM in ICU ("high-risk" group). The primary endpoint evaluated in-hospital major adverse events after unit admission according to VARC-2 criteria.

Results: The mean age was $81.8 \pm 7.5$ years and mean EuroSCORE II was $7.5 \pm 4.8 \%$. In total, 116 patients (25.8\%) were considered as "low-risk" patients and 163 patients (36.3\%) were referred to GCW, including those with immediate pacemaker implantation. A total of 96 patients $(21.3 \%)$ reached the primary endpoint including mainly conductive disorders (12.8\%). No major adverse events, particularly no late severe conductive disorder, occurred in the "low-risk" group (negative predictive value of 100\%). Baseline RBBB $(p<0.01)$, LVEF $<40 \%(p=0.02)$ and "high-risk" group $(p<0.01)$ were predictive of outcomes.

Conclusions: Using rigorous periprocedural selection criteria, patients' admission in GCW without TM can be routinely and safely performed in $1 / 3$ of patients after TAVR.
\end{abstract}

\section{Introduction}

Telemetry monitoring (TM) with or without intensive care unit (ICU) admission is considered as the standard of care after transcatheter

\footnotetext{
* Corresponding author at: Department of Cardiology, Arnaud de Villeneuve Hospital, University of Montpellier, Avenue du doyen Giraud, 34295 Montpellier Cedex 5, France. E-mail address: f-leclercq@chu-montpellier.fr (F. Leclercq).

All authors take responsibility for all aspects of the reliability and freedom from bias of the data presented and their discussed interpretation.
}

aortic valve replacement (TAVR) [1-3]. While improvement of procedure results and TAVR population lower risk profile have been observed in recent studies, conductive disorders remain a major issue after TAVR [4-7]. Selection of patients requiring electrocardiogram (ECG) monitoring according to the risk of conductive disorders was recently pointed out by Toggweiller et al. [2]. Feasibility and safety of a selective TM and ICU admission strategy according to the risk of post-procedure adverse events were also shown by our team in 2015 [8]. Recently developed Fast track protocols generally include TM for $12-24 \mathrm{~h}$ [3]. A strategy of selective TM after TAVR according to the risk of adverse 
events, and particularly the risk of conductive disorders, may be proposed to limit TM units and ICU overload in high volume TAVR centers, allowing admission of low-risk patients in general cardiology ward (GCW) without TM. We can also expect shorter hospitalization duration, with potential economic impact, in line with the recent trend to develop algorithms for fast track procedures [3].

We aimed to evaluate the feasibility and safety of TAVR without systematic post procedural TM through an all comer's prospective study.

\section{Methods}

\subsection{Study population}

All patients undergoing TAVR in Montpellier University Hospital (France) between January and December 2017 were prospectively included. TAVR was indicated for severe symptomatic stenosis confirmed by transthoracic echocardiography (TTE) after Heart Team decision regarding to high surgical risk or for inoperable patients. All patients who died before unit admission or were referred to a non-cardiologic unit were excluded from the analysis.

\subsection{Procedural aspects}

All patients underwent TAVR procedure with new-generation balloon-expandable Edwards Sapien 3 (Edwards Lifesciences, Irvine, California) or self-expandable CoreValve Evolut R devices (Medtronic, Inc., Minneapolis, Minnesota). All procedures were performed under general anesthesia or local anesthesia with sedation. The transfemoral access was performed using percutaneous closure (Proglide) or surgical approach as previously described [9]. Alternative subclavian and transcarotid accesses were considered only in case of unsuitable iliofemoral anatomy. No arterial or central venous lines were used. A temporary pacemaker was inserted via the controlateral femoral vein in all patients. Pre-dilatation of the aortic valve was performed according to operator's choice. The optimal position of the valve was checked by fluoroscopy and a rapid pacing (160-200 beats/min) was triggered during the implantation of balloon-expandable prosthesis as previously described [10]. A final control of the procedural result was performed by aortography and TTE. The temporary pacemaker was removed immediately at the end of the procedure in the absence of conduction disorders.

\subsection{Risk profile algorithm and GCW admission}

Patients were divided into 2 groups: "low-risk" and "high-risk" groups, according to pre-defined pre, per and post-procedural criteria. "Low-risk" criteria are presented in Table 1 and were related to patient profile, hemodynamic stability and risk of conductive disorders [8]. Although the previous generation self-expandable Corevalve prosthesis (Medtronic, Inc., Minneapolis, Minnesota) was considered as a "highrisk" criterion for conductive disorder [8], the Evolut $\mathrm{R}$ prosthesis targeting an optimal positioning with low implantation depth $(<4 \mathrm{~mm}$ ) and a "direct" implantation (without predilatation), was no longer considered as a "high-risk" criterion [11,12]. General anesthesia was not considered as a "high-risk" criterion.

Patients included in the "low-risk" group were monitored for $1 \mathrm{~h}$ after the procedure in an anesthesia recovery room, based on our local algorithm, with a TTE performed within the first hour after the procedure. In the absence of immediate post-procedural events, patients were then referred to GCW without TM.

The final clinical decision to transfer the patient to ICU with TM was left to the physician, even if the patient was initially considered at "lowrisk", he was then reclassified at "high-risk" according to "medical decision". "Medical decision" criterion was the final decision to classify the patient as "low-risk" or "high risk" by the referral physician according to his final examination 1-h post-TAVR monitoring. In case of persistent completeatrioventricular (AV) blockat the end of 1-h observation period,
Table 1

Predefined criteria for high versus low-risk groups of patients.

\begin{tabular}{|c|c|c|}
\hline & $\begin{array}{l}\text { Low-risk } \\
\text { group }\end{array}$ & $\begin{array}{l}\text { High-risk } \\
\text { group }\end{array}$ \\
\hline \multicolumn{3}{|l|}{ Before the procedure } \\
\hline Preexisting pulmonary disease with oxygen dependence & - & + \\
\hline Hemodynamic state & Stable & Unstable $^{a}$ \\
\hline Complete right bundle branch block (QRS >120 ms) & - & + \\
\hline Left ventricular ejection fraction $>40 \%$ & + & - \\
\hline Systolic arterial pulmonary pressure $<60 \mathrm{mmHg}^{\mathrm{b}}$ & + & - \\
\hline Transfemoral approach & + & - \\
\hline \multicolumn{3}{|l|}{ During and $1 \mathrm{~h}$ after the procedure } \\
\hline Hemodynamic state & Stable & Unstable \\
\hline Thrombotic/embolic complications & - & + \\
\hline $\begin{array}{l}\text { Major vascular complications (according to VARC } 2 \\
\text { definitions) }\end{array}$ & - & + \\
\hline New conduction disorders ${ }^{c}$ & - & + \\
\hline New atrial or ventricular arrhythmia & - & + \\
\hline $\begin{array}{l}\text { Good positioning of the prosthesis and aortic } \\
\text { regurgitation } \leq 2\end{array}$ & + & - \\
\hline Medical decision $^{d}$ & - & + \\
\hline \multicolumn{3}{|c|}{$\begin{array}{l}\text { a Mean blood pressure }<65 \mathrm{mmHg} \text {, need for hemodynamic support or fluid infusion. } \\
\text { b Assessed by transthoracic echocardiography. } \\
\text { c Any new QRS enlargement }>4 \mathrm{~ms}, \mathrm{QRS}>120 \mathrm{~ms} \text {, any degree of atrio-ventricular block } \\
\text { including fascicular block. } \\
\text { "Medical decision was the final decision to classify the patient as "low-risk" or "high } \\
\text { risk" by the referral physician according to his final examination } 1 \text {-h post-TAVR } \\
\text { monitoring. }\end{array}$} \\
\hline
\end{tabular}

immediate pacemaker implantation was considered. "High-risk" patients were admitted in ICU for at least $24 \mathrm{~h}$ after the procedure. The ICU is a 16 -bed unit with a full-time staff comprising 6 certified physicians and $1 / 3.8$ nurse per patient ratio. The ICU in our center is the only cardiology unit allowing 7/24 continuous TM and including hemodynamic support and noninvasive ventilation assistance. The GCW is a standard cardiology unit, not allowing TM and with $1 / 10.7$ nurse per patient ratio (1/32 overnight). During hospital stay, ECG was recorded at least daily in the 2 groups. New conduction disorder collected included new QRS enlargement $>4 \mathrm{~ms}, \mathrm{QRS}>120 \mathrm{~ms}$ and any degree of AV block or fascicular block. TTE was performed systematically before ICU or GCW admission including LVEF, mean aortic gradient, aortic regurgitation and the presence of pericardial effusion assessment. TTE was also performed before hospital discharge in all patients.

\subsection{Data assessment and ethics regulation}

All patients were given full study information and written consent was collected. No additional testing or biological samples were specifically required for the study. The protocol was approved by the local ethics committee (Comité de Protection des Personnes Sud Méditerranée, Montpellier, France, ID RCB: IRB-MTP_2020_04_202000416), the institutional regulatory authorities and was conducted according to the principals of the 1975 Declaration of Helsinki.

\subsection{Endpoints}

The primary endpoint was a safety combined endpoint including major complications after unit admission (death, stroke, heart failure, pericardial effusion requiring treatment, major bleeding, major vascular complications, myocardial infarction and pacemaker implantation) according to Valve Academic Research Consortium 2 (VARC-2) criteria, in both "low-risk" and "high-risk" groups [13]. Predictive factors of inhospital complications, minor vascular complications, minor bleeding and hospitalization duration were also evaluated in the 2 groups of patients. 


\subsection{Statistical analysis}

Patients' characteristics are presented using mean \pm SD for continuous variables and frequencies and proportions for categorical variables. We compared both groups ("high-risk" versus "low-risk") using Student's test or Mann-Whitney Wilcoxon rank test for continuous variables, depending on the normality of each variable's distribution as attested by a Shapiro-Wilk test, and chi square or Fisher exact test for categorical variables. Multivariate analysis using logistic regression was performed to determine the relative importance of the preprocedural and peri procedural covariates on the occurrence of major complications VARC-2 criteria. Univariate analyses were first executed, by comparing the variables among the two groups (major complication vs no major complication) using Wilcoxon-Mann-Whitney or chisquare or Fisher tests. Then, the variables with a $p$-value lower than 0.15 were considered for a multivariate logistic regression, and the variables with a p-value lower than 0.05 after a stepwise selection of variable were considered statistically significant. 95\% confidence intervals (CIs), Odds ratios (ORs) and p-values were calculated. The goodnessof-fit of the models was assessed using the Hosmer and Lemeshow chi-square test. Statistical analyses were performed using SAS, version 9.1 (SAS Institute, Cary, North Carolina).

\section{Results}

\subsection{Baseline and procedural characteristics}

A total of 453 patients underwent TAVR procedure within the study period and 449 patients were included in the analysis (Fig. 1). The mean age was $81.8 \pm 7.5$ years, 235 patients ( $52.5 \%$ ) were male and the mean Euroscore II was $7.5 \pm 4.8 \%$ (Table 2). A total of 50 patients $(11.1 \%$ ) had previous pacemaker at baseline, mostly in the "low-risk" group $(n=28$, $24.1 \%$ ) (Table 2). Transfemoral access was performed in 410 patients (91.8\%) and a self-expandable device was implanted in 265 patients (59.4\%) (Supplemental Table 1).

A total of 116 patients (25.8\%) were classified as "low-risk" patients. Baseline characteristics in both groups are presented in Table 2.

\subsection{GCW and ICU admission}

In the "low-risk" group, $100 \%$ of patients $(n=116)$ were referred to GCW after the procedure. In the "high-risk" group, 286 patients $(85.9 \%)$ were referred to ICU mainly for TM regarding the risk of conductive disorder and 47 patients (14.1\%) were referred to GCW according to medical decision, mainly related to immediate pacemaker implantation $(n=26)$ (Fig. 1$)$. Details on the 47 patients referred to the GCW despite a "high-risk" profile are presented in Supplemental Table 2 .

\subsection{Primary endpoint}

In-hospital adverse events occurred in 129 patients (28.7\%) including 96 (21.3\%) reaching the primary endpoint of major complications after unit admission, all occurring in "high-risk" patients (Fig. 1 and Table 3). Major adverse events occurred in 33 patients (7.3\%) during the procedure including 29 high-degree AV block (6.5\%) requiring pacemaker implantation, 2 prosthesis migration $(0.4 \%)$ and 2 left ventricle perforation $(0.4 \%)$. In-hospital pacemaker implantation was observed in 86 patients (19.1\%) including 3 patients $(2.6 \%)$ in the "low-risk" group before unit admission and 83 patients $(24.9 \%)$ in the "high-risk" group. Pacemaker implantation was not related to prosthesis type $(n=37$ with Sapien 3 device (20.4\%) versus 49 with Evolut $\mathrm{R}$ device (18.5\%), respectively, $p=$ 0.8 ). The negative predictive value for complications of the "lowrisk" group classification was 100\% (95\% Confidence Interval: $100-100)$. In the 47 patients from the "high-risk" group referred to the GCW, 3 patients (6.4\%) had major complications including 2 pacemaker implantations (4.3\%) in patients with enlargement of previous incomplete LBBB and 1 cardiac tamponade after pacemaker implantation leading to ICU transfer and death.

\subsection{Secondary endpoints}

All patients with minor vascular complications or minor bleedings had favourable evolution and did not require secondary transfer to ICU. Hospitalization duration was shorter in "low-risk" compared to

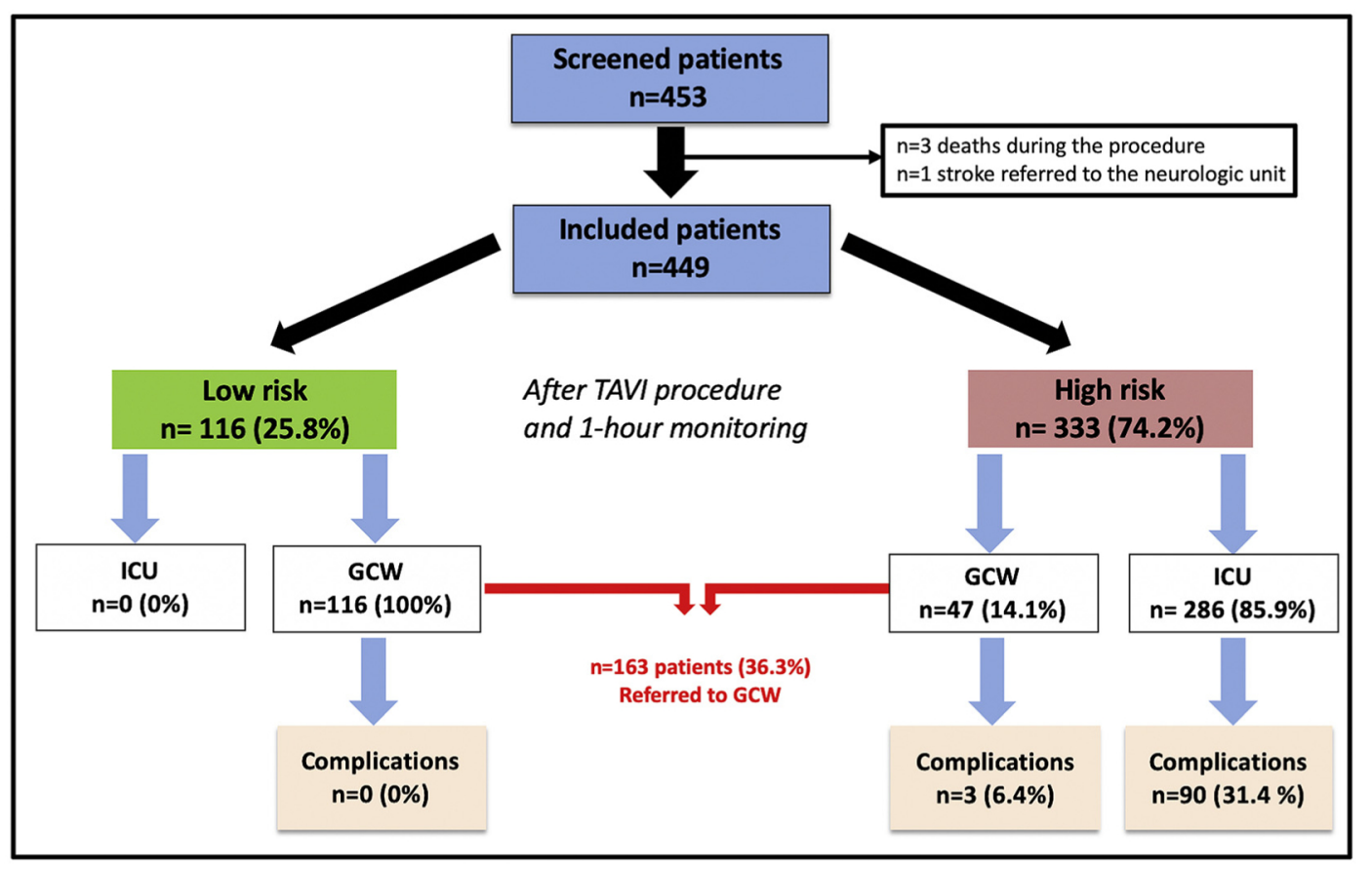

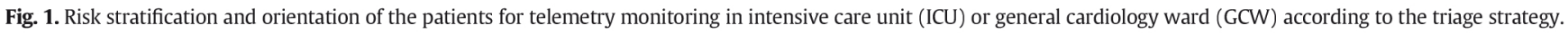


Table 2

Baseline characteristics.

\begin{tabular}{|c|c|c|c|c|}
\hline Variables & Total population $n=449$ & Low risk group $n=116$ (25.8) & High risk group $n=333$ (74.2) & $p$-value \\
\hline Age (years), mean \pm SD & $81.8 \pm 7.5$ & $82.7 \pm 6.4$ & $81.5 \pm 7.7$ & 0.2 \\
\hline Male gender, n(\%) & $235(52.5)$ & $68(58.6)$ & $167(50.3)$ & 0.2 \\
\hline Body Mass Index $(\mathrm{kg} / \mathrm{m} 2)$, mean $\pm \mathrm{SD}$ & $25.9 \pm 4.4$ & $26.0 \pm 4.0$ & $25.9 \pm 4.6$ & 0.06 \\
\hline Euroscore II (\%),mean \pm SD & $7.5 \pm 4.8$ & $6.5 \pm 3.5$ & $7.8 \pm 5.1$ & 0.04 \\
\hline Hypertension, $\mathrm{n}(\%)$ & $302(67.3)$ & $75(64.7)$ & $227(68.2)$ & 0.7 \\
\hline Diabetes mellitus, n(\%) & 134 (29.9) & $26(22.6)$ & $108(32.4)$ & 0.05 \\
\hline Active smoker, $\mathrm{n}(\%)$ & $34(7.6)$ & $10(8.7)$ & $24(7.2)$ & 0.6 \\
\hline Severe pulmonary disease, $\mathrm{n}(\%)$ & $15(3.3)$ & $0(0)$ & $15(4.5)$ & 0.01 \\
\hline Systolic PAP > 60 mmHg, $\mathrm{n}(\%)$ & $4(0.9)$ & $0(0)$ & $4(1.2)$ & 0.5 \\
\hline Coronary artery disease/stroke, n(\%) & $247(55.1)$ & $68(58.6)$ & $179(53.8)$ & 0.4 \\
\hline Peripheral artery disease, $n(\%)$ & $36(8.0)$ & $7(6.0)$ & $29(8.7)$ & 0.4 \\
\hline Atrial fibrillation, $\mathrm{n}(\%)$ & $150(33.4)$ & $31(26.7)$ & $119(35.7)$ & 0.08 \\
\hline Cirrhosis, $\mathrm{n}(\%)$ & $7(1.6)$ & $0(0)$ & $7(2.1)$ & 0.1 \\
\hline Previous Pacemaker, n(\%) & $50(11.1)$ & $28(24.1)$ & $22(6.6)$ & $<0.0001$ \\
\hline \multicolumn{5}{|l|}{ Admission electrocardiogram } \\
\hline First degree AVB, n(\%) & $83(18.5)$ & $17(14.7)$ & $66(19.8)$ & 0.2 \\
\hline Complete LBBB, $\mathrm{n}(\%)$ & $47(10.5)$ & $8(6.9)$ & $39(11.7)$ & 0.1 \\
\hline Complete RBBB, $\mathbf{n}(\%)$ & $52(11.6)$ & $\mathbf{0}(\mathbf{0})$ & $52(15.6)$ & $<0.0001$ \\
\hline \multicolumn{5}{|l|}{ Treatment } \\
\hline VKA, n(\%) & $74(16.5)$ & $16(13.9)$ & $58(17.4)$ & 0.4 \\
\hline Dual antiplatelet, $\mathrm{n}(\%)$ & $115(25.6)$ & $40(34.5)$ & $75(22.5)$ & 0.01 \\
\hline Direct oral anticoagulants, $\mathrm{n}(\%)$ & $54(12.0)$ & $10(8.6)$ & $44(13.2)$ & 0.2 \\
\hline Simple antiplatelet + VKA, n(\%) & $38(8.5)$ & $6(5.2)$ & $32(9.6)$ & 0.1 \\
\hline Left ventricular ejection fraction & & & & 0.0018 \\
\hline$<30 \%, \mathrm{n}(\%)$ & $11(2.5)$ & $0(0)$ & $11(3.3)$ & \\
\hline $30-40 \%, n(\%)$ & $28(6.2)$ & $0(0)$ & $28(8.4)$ & \\
\hline$>40 \%, \mathrm{n}(\%)$ & $410(91.3)$ & $116(100)$ & $294(88.3)$ & \\
\hline \multicolumn{5}{|l|}{ Biological data } \\
\hline Creatinine clearance $(\mathrm{mL} / \mathrm{min})$, mean $\pm \mathrm{SD}$ & $53 \pm 18.3$ & $55.2 \pm 17.1$ & $52.1 \pm 18.7$ & 0.1 \\
\hline Hemoglobin $(\mathrm{g} / \mathrm{dL})$, mean \pm SD & $12.5 \pm 1.7$ & $12.8 \pm 1.5$ & $12.4 \pm 1.7$ & 0.04 \\
\hline
\end{tabular}

*AVB: atrio-ventricular block, †LBBB: left bundle block branch; †RBBB: right bundle block branch; §PAP: pulmonary artery pressure, ||VKA: vitamin K antagonist.

Table 3

In-hospital complications according to VARC-2 criteria after unit transfer decision.

\begin{tabular}{|c|c|c|c|c|}
\hline Variables & Total population $n=449$ & Low-risk group $n=116(25.8)$ & High-risk group $n=333(74.2)$ & $p$ value \\
\hline Unstable hemodynamic, n (\%) & $4(0.9)$ & $0(0)$ & $4(1.2)$ & 0.6 \\
\hline Pericardial effusion requiring treatment, $\mathrm{n}(\%)$ & $6(1.3)$ & $0(0)$ & $6(1.8)$ & 0.3 \\
\hline Ventricular arrhythmia, n (\%) & $3(0.7)$ & $0(0)$ & $3(0.9)$ & 0.6 \\
\hline Death from any cause, $\mathrm{n}(\%)$ & $3(0.7)$ & $0(0)$ & $3(0.9)$ & 0.6 \\
\hline Acute pulmonary edema, $\mathrm{n}(\%)$ & $3(0.7)$ & $0(0)$ & $3(0.9)$ & 0.6 \\
\hline Major bleeding, $\mathrm{n}(\%)$ & $4(0.9)$ & $0(0)$ & $4(1.2)$ & 0.6 \\
\hline Major vascular complications, n (\%) & $8(1.8)$ & $0(0)$ & $8(2.4)$ & 0.2 \\
\hline Stroke, n (\%) & $2(0.5)$ & $0(0)$ & $2(0.6)$ & 0.9 \\
\hline Pacemaker implantation after unit transfer ${ }^{\mathrm{a}}(\%)$ & $57(12.8)$ & $0(0)$ & $57(17.3)$ & $<0.0001$ \\
\hline Myocardial infarction, n (\%) & $0(0)$ & $0(0)$ & $0(0)$ & NA \\
\hline Acute kidney injury, n (\%) & $5(1.1)$ & $0(0)$ & $5(1.5)$ & 0.3 \\
\hline Secondary transfer to intensive care unit, $\mathrm{n}(\%)$ & $1(0.2)$ & $0(0)$ & $1(0.3)$ & 0.6 \\
\hline Total, n (\%) & $96(21.3)$ & $\mathbf{0}(\mathbf{0})$ & $96(28.8)$ & $<0.05$ \\
\hline
\end{tabular}

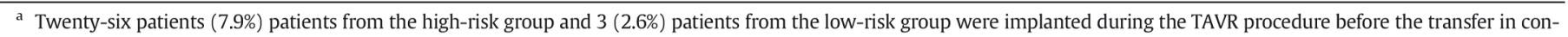
ventional care unit (not collected in this table).

"high-risk" patients ( $3.4 \pm 3.7 v s 4.2 \pm 3.4$ days, respectively, $p<0.0001)$ and for GCW compared to ICU patients ( $2.8 \pm 1.9 \mathrm{vs} 4.4 \pm 4.1$ days respectively, $p<0.0001$ ). Median ICU length of stay was $1.8 \pm 1.8$ days. Minor changes on daily ECG including first degree AV block and incomplete LBBB (QRS enlargement $>4 \mathrm{~ms}$ and QRS duration $<120 \mathrm{~ms}$ ) were highlighted in 8 patients (1.8\%) and 24 patients (5.3\%) respectively, all in the "high-risk" group. None of these minor changes required further investigation or delayed pacemaker implantation. At one month follow-up, only 1 "high-risk" patient ( $0.2 \%)$ underwent pacemaker implantation after hospital discharge for complete LBBB associated with dyspnea.

\subsection{Predictive factors of outcomes}

In multivariate analysis, pre-procedural predictive factors of in-hospital complications were $\mathrm{RBBB}$ at admission ( $\mathrm{OR}=3.6[95 \% \mathrm{CI}=1.64-1.79]$; $p<0.01)$, LVEF $<40 \%(\mathrm{OR}=10.11[95 \% \mathrm{CI}=1.32-77.13] ; p=0.02)$ and "high-risk" group $(\mathrm{OR}=13.38[95 \% \mathrm{Cl}=3.15-56.76] ; p<0.01$.

\section{Discussion}

In this prospective study, we aimed to confirm the feasibility and safety of TAVR without systematic TM and ICU admission in selected patients with 3 main findings:

1/ One third of patients may be admitted safely in GCW without continuous TM after TAVR.

2/ Pre, per and post-procedure low risk criteria may predict the absence of complications, particularly for severe conductive disorders, with a $100 \%$ negative predictive value.

3 / Inclusion in the "low-risk" group and avoiding TM or ICU admission were associated with shorter hospitalization duration. 


\subsection{Proportion of patients that may avoid ICU}

In this study, the negative predictive value for complications in "lowrisk" patients substantially improved compared to our previous experience (8). This may be explained by a more "aggressive" classification of patients as "high-risk" in the presence of any new conduction disorder. Similarly, most peri-procedural complications were reported as occurring before patient's unit admission, particularly regarding to highdegree conduction disorders [2,14]. Furthermore, according to Toggweiller et al., no delayed conduction disorder occurred in patients with normal post-procedural ECG [2]. Thus, as conduction disorder remains the main issue after TAVR, patients with normal post-procedure ECG, around $1 / 3$ in our study, may not require TM after TAVR as previously observed by Toggweiller et al. [2]. By adding a "medical decision" criterion for risk stratification, our study increased patients' selection to avoid safely both TM and ICU. While in our center TM is feasible only in ICU, TM without ICU may also be considered only in patients at high-risk for conduction disorders. Only one study, assessing early discharge after TAVR,pointed out the feasibility of avoiding ICU in $12.6 \%$ of patients referred directly to GCW after TAVR [15]. However, no admission criteria were described in this study to predict ICU admission and the rate of patients avoiding ICU or intermediate care unit was lower than in the present study. Considering patients initially at high-risk for conductive disorders, undergoing immediate post-TAVR pacemaker implantation $(n=26)$, proportion of patients that could be admitted directly in GCW represent more than $1 / 3$ of our population. While more than a half of patients with persistent AV block at the time of pacemaker implantation after TAVR were reported to have pacemaker dependency, immediate pacemaker implantation may be considered in these patients with persistent high-degree AV block one-hour post-TAVR [16].

\subsection{Post-procedural complications regarding to the risk group}

Pacemaker implantation for new conduction disorder occurring in $19.1 \%$ of patients was the main complication following TAVR, as previously described $[17,18]$. This high rate of pacemaker implantation observed in our study may be explained by first large indications including left bundle branch block $>130 \mathrm{~ms}$ and second, our aging population. Moreover, adverse events occurred only in "high-risk" patients and pacemaker implantations were required mainly in this group. Indeed, RBBB at admission, previously reported as the main predictive factor for conduction disorders, was a "high-risk" criterion in the present study $[2,17,18]$. On the other hand, major bleeding and vascular complications were rare in our study. Indeed, new generation devices, better patients' selection, increased operators experience allowed to dramatically decrease these complications. Interestingly, all complications only occurred in the "high-risk" group of patients. These data suggest that our algorithm including simple admission, per and 1-h postprocedure criteria may be relevant in clinical practice to identify patients at risk of complications.

\subsection{Hospitalization duration}

In our study, hospitalization duration was shorter in patients with a "low-risk" profile. Previous reports, consistent with our findings, pointed out the relationship between in-hospital complications and hospitalization duration $[15,19]$. However, in our study, the absence of ICU admission may also contribute by itself to earlier discharge in these patients, although this should be confirmed in a randomized study. Economic impact of both avoiding TM and/or ICU admission and reduction of hospitalization duration may also be evaluated specifically. Considering the different approaches proposed to reduce hospitalization duration after TAVR with fast track protocols [3,15], a simple risk stratification including the need of TM as proposed in our study may be a new efficient strategy.

\subsection{Study limitations}

The first limitation is the single-center design of this study not allowing to extend our results to all centers, however algorithm criteria are simple and can be assessed easily. The second limit is that risk stratification criteria were based on our experience and literature but have not been validated in randomized studies. Third, one of our criteria, "medical decision" remains subjective but shows that algorithm may integrate medical experience. Finally, despite the negative predictive value of $100 \%$ for late-onset conduction disorders, the sample size and the rate of events may be too small to be generalizable. However, considering that longer monitoring up to 7 days in hospital may not be generalizable in all TAVR patients, the strategy proposed in our study during hospital stay seems relevant. The interest of out of hospital monitoring systems as implantable events recorders could be specifically evaluated .

\section{Conclusion}

Our results confirm that, with a rigorous patients' selection, TAVR can be routinely and safely performed without systematic TM and ICU admission in at least $1 / 3$ of patients. Conductive disorders remain the main adverse events and may be evaluated by rigorous ECG analysis before, within and after the procedure. This strategy may be complementary to cost-effective approaches aiming to shorten hospitalization duration in selected patients.

\section{Disclosures}

F. Leclercq received research grants from Edwards, Medtronic, Boehringer; consultant fees from Boehringer; and lecture fees from Astra Zeneca and Bayer.

B. Lattuca received research grants from ACTION Study group, Biotronik, Boston Scientific, Daiichi-Sankyo, Fédération Française de Cardiologie and Institute of CardioMetabolism and Nutrition; consultant fees from Daiichi-Sankyo and Eli Lilly; and lecture fees from AstraZeneca and Novartis.

$\mathrm{G}$ Cayla received research grants/consultant fees/lectures fees from Amgen, AstraZeneca, Bayer, Boehringer-Ingelheim, Boston, Biotronik, Bristol-Myers Squibb, Daiichi-Sankyo, Eli-Lilly, Europa, Fédération Française de Cardiologie, Fondation Cœur \& Recherche, Medtronic, MSD, Pfizer, Sanofi-Aventis.

M. Akodad received research grants from Biotronik, Edwards Lifescience and Medtronic.

P. Robert received research grants from Edwards Lifescience.

\section{Sources of funding}

None.

\section{Acknowledgments}

None.

\section{References}

[1] H. Baumgartner, V. Falk, J.J. Bax, et al., 2017 ESC/EACTS guidelines for the management of valvular heart disease, Eur. Heart J. 38 (2017) 2739-2791.

[2] S. Toggweiler, S. Stortecky, E. Holy, et al., The electrocardiogram after Transcatheter aortic valve replacement determines the risk for post-procedural high-degree AV block and the need for telemetry monitoring, JACC Cardiovasc. Interv. 9 (2016) 1269-1276.

[3] D.A. Wood, S.B. Lauck, J.A. Cairns, et al., The Vancouver 3M (multidisciplinary, multimodality, but minimalist) clinical pathway facilitates safe next-day discharge 
home at low-, medium-, and high-volume Transfemoral Transcatheter aortic valve replacement centers: the 3M TAVR study, JACC Cardiovasc. Interv. 12 (2019) 459-469.

[4] M.B. Leon, C.R. Smith, M.J. Mack, et al., Transcatheter or surgical aortic-valve replacement in intermediate-risk patients, N. Engl. J. Med. 374 (2016) 1609-1620.

[5] M.J. Reardon, N.M. Van Mieghem, J.J. Popma, et al., SURTAVI investigators. Surgical or Transcatheter aortic-valve replacement in intermediate-risk patients, N. Engl. J. Med. 376 (2017) 1321-1331.

[6] J.J. Popma, G.M. Deeb, S.J. Yakubov, et al., Transcatheter aortic-valve replacement with a self-expanding valve in low-risk patients, N. Engl.J. Med. 380 (2019) 1706-1715

[7] M.J. Mack, M.B. Leon, V.H. Thourani, et al., Transcatheter aortic-valve replacement with a balloon-expandable valve in low-risk patients, N. Engl. J. Med. 380 (18) (2019) 1695-1705.

[8] F. Leclercq, A. Iemmi, B. Lattuca, et al., Feasibility and safety of Transcatheter aortic valve implantation performed without intensive care unit admission, Am. J. Cardiol. 118 (2016) 99-106.

[9] F. Leclercq, M. Akodad, J.-C. Macia, et al., Vascular complications and bleeding after Transfemoral Transcatheter aortic valve implantation performed through open surgical access, Am. J. Cardiol. 116 (2015) 1399-1404.

[10] F. Nijhoff, P. Agostoni, M. Samim, et al., Optimisation of transcatheter aortic balloonexpandable valve deployment: the two-step inflation technique, Eurolntervention. 9 (2013) 555-563.

[11] H. Thiele, T. Kurz, H.-J. Feistritzer, et al., General versus local anesthesia with conscious sedation in Transcatheter aortic valve implantation: the randomized SOLVE-TAVI trial, Circulation 142 (15) (2020) 1437-1447.
[12] J. Webb, D. Wood, J. Sathananthan, U. Landes, Balloon-expandable or selfexpandable transcatheter heart valves. Which are best? Eur. Heart J. 41 (20) (2020) 1900-1902.

[13] A.P. Kappetein, S.J. Head, P. Généreux, et al., Valve academic research Consortium-2. Updated standardized endpoint definitions for transcatheter aortic valve implantation: the valve academic research Consortium-2 consensus document, J. Thorac. Cardiovasc. Surg. 145 (2013) 6-23.

[14] G.C.M. Siontis, P. Jüni, T. Pilgrim, et al., Predictors of permanent pacemaker implantation in patients with severe aortic stenosis undergoing TAVR: a meta-analysis, J. Am. Coll. Cardiol. 64 (2014) 129-140.

[15] M. Barbanti, M.S. van Mourik, M.S. Spence, et al., Optimising patient discharge management after transfemoral transcatheter aortic valve implantation: the multicentre European FAST-TAVI trial, Eurolntervention. 15 (2019) 147-154.

[16] R.M. Kaplan, A. Yadlapati, E.P. Cantey, et al., Conduction recovery following pacemaker implantation after transcatheter aortic valve replacement, Pacing Clin. Electrophysiol. 42 (2019) 146-152.

[17] M. Barbanti, S. Gulino, G. Costa, C. Tamburino, Pathophysiology, incidence and predictors of conduction disturbances during Transcatheter aortic valve implantation, Expert. Rev. Med. Devices. 14 (2017) 135-147.

[18] H. Kaneko, F. Hoelschermann, M. Seifert, et al., Predictors of permanent pacemaker implantation after transcatheter aortic valve implantation for aortic stenosis using Medtronic new generation self-expanding CoreValve Evolut R, Heart Vessel. 34 (2019) 360-367.

[19] E. Durand, H. Eltchaninoff, A. Canville, et al., Feasibility and safety of early discharge after transfemoral transcatheter aortic valve implantation with the Edwards SAPIEN-XT prosthesis, Am. J. Cardiol. 115 (2015) 1116-1122. 